\title{
Trombectomía quirúrgica en trombosis aórtica neonatal asociada a deshidratación hipernatrémica
}

\section{Surgical thrombectomy in neonatal aortic thrombosis associated with hypernatremic dehydration}

\author{
Selene Herrera-Martínez, Alejandro Celis-Jiménez*, Ana B. Calderón-Alvarado y Vanessa Huerta-Álvarez \\ Departamento de Angiología y Cirugía Vascular, Instituto Mexicano del Seguro Social, Guadalajara, Jalisco, México
}

\begin{abstract}
Resumen
Se realizó una revisión de la literatura de 2005 a 2020 de los casos de trombosis aórtica neonatal asociada a deshidratación hipernatrémica, y se comparó con la presentación clínica de dos casos en nuestro centro médico, los cuales se manifestaron con isquemia irreversible de las extremidades. En la literatura se encontraron cinco casos, de los cuales uno tuvo desenlace fatal. Se realizó trombectomía mecánica en ambos casos y se anticoaguló durante el posquirúrgico de forma sistémica, ambos manejos con adecuada evolución. A pesar de que aún no existe piedra angular en el abordaje y tratamiento de la trombosis aórtica neonatal, actualmente existen opciones terapéuticas en evaluación como la anticoagulación, la trombólisis sistémica, la trombólisis dirigida por catéter y la trombectomía mecánica; en nuestro caso, se optó por el manejo abierto debido a la gravedad y agudeza de ambos escenarios clínicos.
\end{abstract}

Palabras clave: Trombosis aórtica neonatal espontánea. Deshidratación hipernatrémica. Trombectomía abierta.

\begin{abstract}
We conducted a review of published cases between 2005 to 2020, on neonatal acute aortic thrombosis associated with hypernatremic dehydration, to compare them with the clinical presentation of two cases in our Medical Center, manifested with irreversible ischemia of the extremities. Five cases were found in the literature, one had a fatal outcome. Mechanical thrombectomy was performed in both patients, and postoperatively they were placed on systemic anticoagulation, both progressed favorably. Although there is still no cornerstone in the optimal approach and management of neonatal aortic thrombosis, currently there are therapeutics options under study, such as anticoagulation, systemic thrombolysis, catheter-directed thrombolysis and mechanical thrombectomy. In our experience, open surgical management was opted due to the severity, clinical status and acuteness of both clinical scenarios.
\end{abstract}

Key words: Spontaneous neonatal aortic thrombosis. Hypernatremic dehydration. Surgical thrombectomy.

Correspondencia:

*Alejandro Celis-Jiménez

E-mail: celisj3@ hotmail.com bajo la licencia CC BY-NC-ND license (http://creativecommons.org/licenses/by-nc-nd/4.0/).
Disponible en internet: 24-09-2021 Rev Mex Angiol. 2021;49(3):93-99 www.RMAngiologia.com 


\section{Introducción}

La presentación de trombosis aórtica neonatal se reporta con muy poca frecuencia en la literatura contemporánea. Entre las causas más reconocidas se presenta el cateterismo de la arteria umbilical como la principal, a la que se agregan procedimientos secundarios como infusión de calcio por el catéter umbilical, colocación del catéter arriba de las arterias renales y uso de un catéter 3.5 $\mathrm{Fr}^{1}$. No constan datos establecidos con respecto a una tipificación de la trombosis aórtica espontánea, sin embargo, en un artículo publicado en 2010 se hace referencia a una sugerencia de clasificación hecha en 1992 que incluyó clínica, englobando aspectos locales y sistémicos (Tabla 1) ${ }^{1}$.

En el rango de tiempo estudiado, se encontraron cinco casos de trombosis aórtica abdominal secundaria a deshidratación hipernatrémica, de los cuales uno tuvo un desenlace fatal ${ }^{2}$. Sin embargo, no hay un protocolo de tratamiento y seguimiento del paciente, además en ninguno de los casos analizados existen datos de regresión espontánea de la trombosis, por lo que se necesitan más líneas de investigación en esta población.

Por lo anterior, el objetivo de este trabajo es describir el tratamiento quirúrgico de los casos de neonatos enviados de unidad de segundo nivel, previamente sanos, sin cateterización umbilical; con clínica de isquemia aguda irreversible de extremidad inferior, cuyo común denominador fue la deshidratación hipernatrémica; mismos a los que se les realizó una angiotomografía donde se observó trombosis aórtica abdominal, clasificada como mayor.

\section{Métodos}

Se reportan dos casos se trombosis aórtica espontánea en asociación con deshidratación hipernatrémica.

\section{Caso 1}

Presentamos a neonato de sexo masculino de ocho días de vida extrauterina, de término de 39 semanas, eutrófico, hijo de madre diabética y preeclámpsica. Mediante interrogatorio indirecto a madre con control prenatal a partir de primer trimestre.

Inició padecimiento con fiebre de hasta $40{ }^{\circ} \mathrm{C}$, ausencia de evacuaciones, oliguria, hiporexia e irritabilidad manejándolo con antibióticos, a base de ampicilina y amikacina sin mejoría. Dos días posteriores inicia con deshidratación severa, fiebre de $39.6{ }^{\circ} \mathrm{C}$; a la

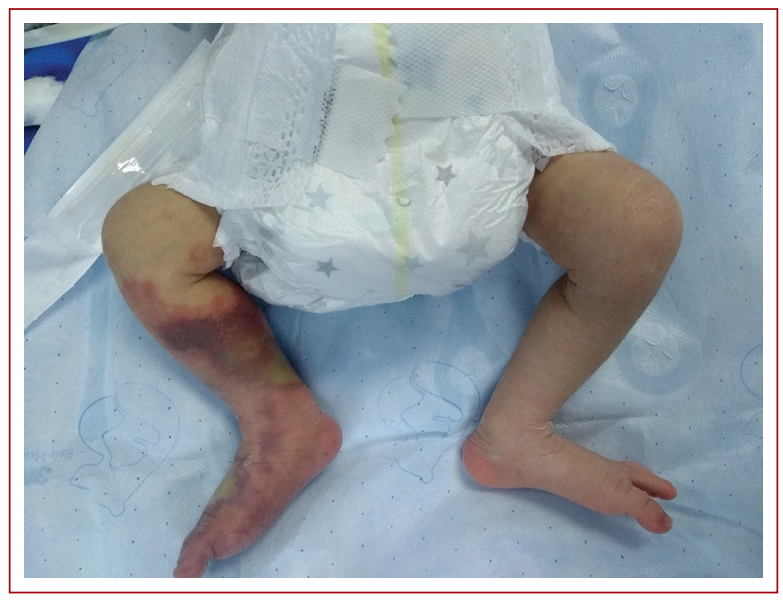

Figura 1. Neonato 8 días con isquemia irreversible de miembro pélvico derecho.

exploración con cianosis en extremidades inferiores con disminución de los pulsos periféricos (Fig. 1).

Se realizó valoración del servicio de angiología, que reportó datos francos de isquemia bilateral de miembros pélvicos, con irreversibilidad de miembro pélvico derecho hasta nivel supracondíleo, por lo que se inició el manejo a base de enoxaparina $1 \mathrm{mg} / \mathrm{kg} / \mathrm{dosis}$. Y se envió al centro de referencia de nuestro servicio.

En exploración física dirigida se encontró extremidad inferior derecha, con hipotermia, lesiones purpúricas de isquemia desde dedos hasta rodilla, no se palparon pulsos y dolor a la palpación en hueco poplíteo, sin reflejos osteotendinosos, pie caído, al Doppler de onda continua con ausencia de flujos de poplítea, tibiales y peronea; pierna izquierda con eutermia, Ilenado capilar 2 segundos, se palparon pulsos desde femoral a pedio, con presencia de flujos trifásicos desde femoral hasta pedio, con adecuada coloración de piel, reflejos osteotendinosos presentes.

Paraclínicos al ingreso con datos de lesión renal aguda, creatinina 1.9, urea 131 ; hipernatremia (fósforo 5.7 ), calcio no ionizado 8.5 , cloro 128 , sodio 168 , potasio 4.5 , magnesio 2.1 ; hemoglobina 18.2 , hematocrito 56.4 , plaquetas 84,000 , leucocitos 17.53 , con desviación a la izquierda; tiempos de coagulación tiempo de protombina (TP) 16, tiempo parcial de tromboplastina (TPT) 47.1, índice internacional normalizado (INR) 1.3, fibrinógeno 37, dímero D 881, lactato deshidrogenasa (DHL) 2056, además con hiperbilirrubinemia multifactorial, fuera de rangos de fototerapia, bilirrubina total (BT) 22.5, por lo que se ajustó aporte de líquidos y soluciones intravenosas; finalmente con elevación de procalcitonina de $2.7 \mathrm{mg} /$ 
Tabla 1. Clasificación clínica de trombosis aórtica neonatal 1992

\begin{tabular}{|l|l|l|}
\hline Grado de trombosis & Manifestaciones locales & Manifestaciones sistémicas \\
\hline Menor & $\begin{array}{l}\text { Disminución del pulso femoral, incapacidad para retirar sangre } \\
\text { del catéter }\end{array}$ & Hipertensión \\
\hline Moderada & $\begin{array}{l}\text { Lo de arriba más: ausencia de pulsos femorales, isquemia } \\
\text { periférica evidenciada por extremidades frías, pálidas y moteadas } \\
\text { y llenado capilar deficiente }\end{array}$ & $\begin{array}{l}\text { Hipertensión más insuficiencia cardiaca } \\
\text { congestiva }\end{array}$ \\
\hline Mayor & $\begin{array}{l}\text { Lo de arriba más: isquemia grave que amenaza las extremidades, } \\
\text { evidenciada por gangrena, pérdida de tejido y/o parálisis }\end{array}$ & $\begin{array}{l}\text { Lo de arriba más: insuficiencia renal, } \\
\text { acidosis con afectación visceral o sepsis }\end{array}$ \\
\hline
\end{tabular}

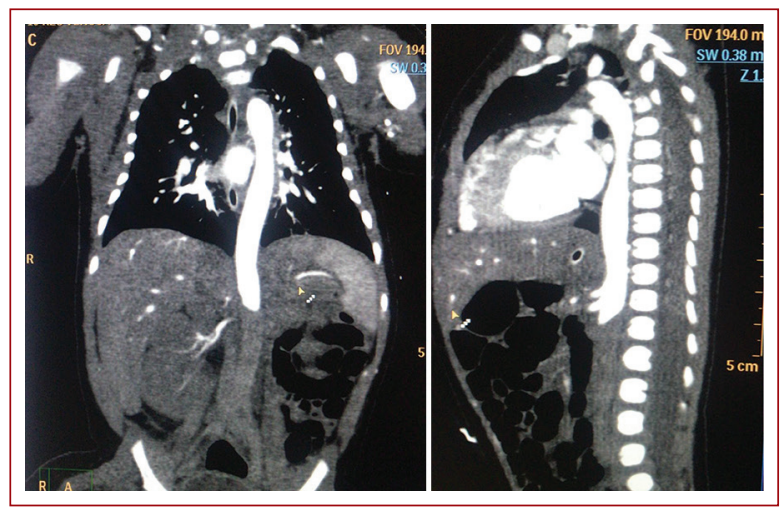

Figura 2. Neonato de 23 días con isquemia irreversible en miembro pélvico derecho.

dl y lactato de $4.8 \mathrm{ml} / \mathrm{dl}$. Se realizó cultivo de orina, sangre central y periférico, sin crecimiento bacteriano cinco días después. Se realizó ecocardiograma transtorácico, resultó conducto arterioso permeable en vías de cierre, foramen oval permeable, función ventricular conservada, sin evidencia de vegetaciones ni trombos.

Se realizó angiotomografía, donde se observó trombosis aórtica total desde segmento infrarrenal, con recanalización distal a nivel femoral (Fig. 2), se ingresó a sala de operaciones el mismo día con carácter de urgencia, bajo anestesia general balanceada, se incidió con abordaje medio supraumbilical e infraumbilical, se disecó de forma roma hasta el origen de las arterias renales, se realizó control vascular de estas y de la aorta de forma individual con cintillas vasculares, se realizó arteriotomía y trombectomía mecánica aórtica, con un catéter Fogarty $3 \mathrm{Fr}$ en tres ocasiones, hasta obtener reflujo arterial. Se encontró: trombosis aórtica yuxtarrenal, con isquemia intestinal de asa fija, lo cual recuperó posterior a trombectomía (Fig. 3), con perfusión adecuada de miembro inferior izquierdo, se decidió cierre posterior a $15 \mathrm{cc}$ de trombos mixtos; durante el

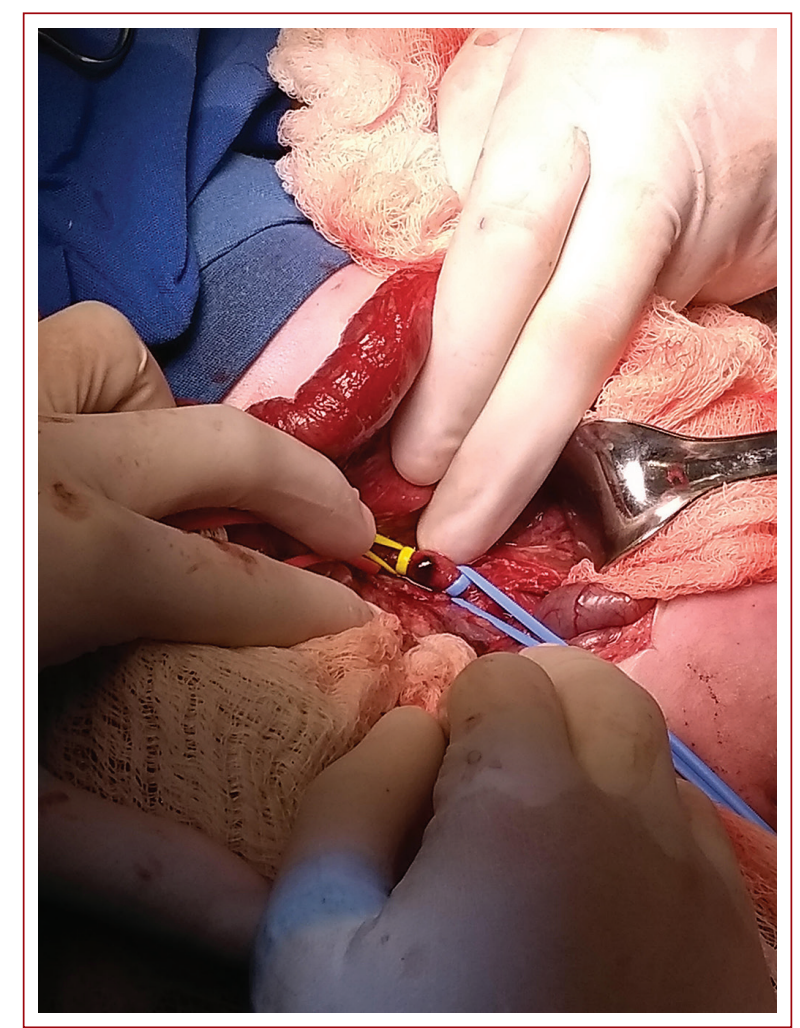

Figura 3. Trombosis aórtica abdominal de neonato de 8 días.

transquirúrgico se necesitó apoyo de aminas vasoactivas, retirándolas por completo en el posquirúrgico inmediato y se inició anticoagulación a base de enoxaparina $1 \mathrm{mg} / \mathrm{kg}$ a las 12 horas de finalizado el procedimiento. El estado vascular posterior a trombectomía no presentó síndrome de reperfusión, fue manejado adecuadamente con hidratación agresiva, sin datos de insuficiencia renal; resto de extremidades íntegras, con adecuada irrigación, flujos trifásicos. Se realizó amputación supracondílea de miembro pélvico derecho por el servicio de traumatología y ortopedia sin complicaciones. Se egresó paciente con mejoría clínica. 




Figura 4. Trombosis aórtica abdominal de neonato de 23 días. Tomografía axial computarizada prequirúrgica (izquierda) y posquirúrgica (derecha).

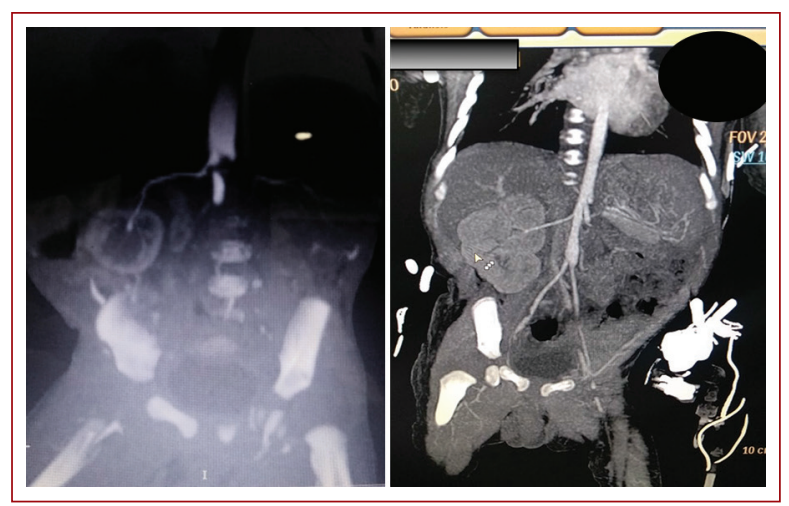

Figura 5. Aorta abdominal realizando trombectomía de neonato de 8 días.

\section{Caso 2}

Paciente de sexo femenino de 23 días de vida extrauterina, producto de segunda gestación, madre y padre negaron enfermedades crónicas degenerativas. Control prenatal desde el $2 .^{\circ}$ mes, niega hipertensión gestacional y diabetes mellitus.

A la semana del nacimiento comenzó con llanto incontrolable, la madre nota cambio de coloración e hipotermia en pie izquierdo, además de hiporexia de 3-4 días de evolución, por lo que acudió a valoración a hospital de su región, donde se observó necrosis de los cinco dedos del pie derecho, por lo que se envió 24 horas después a nuestro centro de referencia.

Se recibió a su ingreso paciente en malas condiciones generales, hipoactivo, mal estado de hidratación, mucosas secas, llanto sin lágrimas, con oxígeno

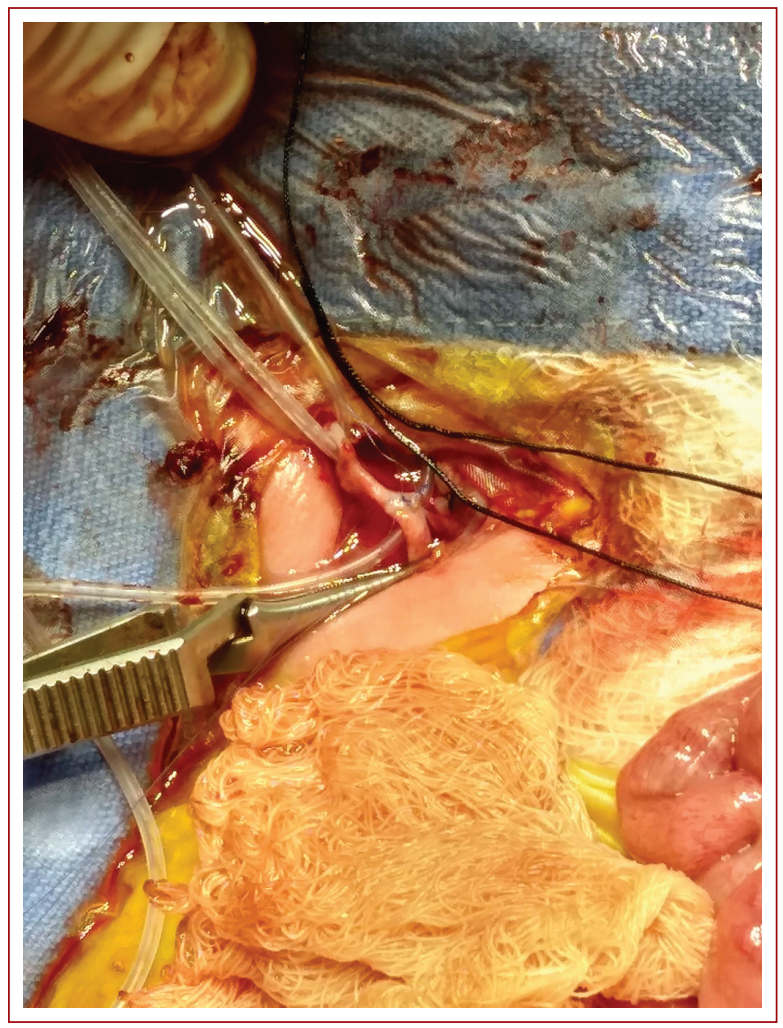

Figura 6. Rafia aórtica después de trombectomía de neonato de 23 días.

complementario en caso cefálico a 3 I por minuto; a la exploración vascular dirigida, extremidades con necrosis inframaleolar de pie izquierdo, no se palparon pulsos (Fig. 4). Extremidad contralateral con pulsos disminuidos de intensidad, llenado capilar de 3 segundos, sin lesiones presentes. Paraclínicos al ingreso, con hemoglobina 18.0, hematocrito 62.9 leucocitos 13,000, tiempos de coagulación: TP 14.7, TTPa 57.4, INR 1.14, con insuficiencia renal aguda evidenciada con urea 301 , creatinina 2.7 y ácido úrico 9.4; finalmente con hipernatremia, con electrolitos séricos de: fósforo 4.8 , calcio 9.7 , cloro 130 , potasio 4.7 , sodio 161 y magnesio 2.5; DHL 3472. Se realizó cultivo sérico y de orina, sin obtener desarrollo bacteriano tras siete días.

Se llevó a cabo manejo de la deshidratación hipernatrémica, se anticoaguló con enoxaparina $1 \mathrm{mg}$ subcutánea cada 12 horas y doble esquema de antibiótico ajustado a función renal (cefotaxima y amikacina, lo cual se retiró posterior a resultado de cultivo). Durante su estancia en urgencias presentó deterioro ventilatorio, por lo que se procedió a intubación orotraqueal sin uso de aminas vasoactivas. 
Tabla 2. Casos publicados de 2005-2020 de trombosis aórtica espontánea neonatal secundaria a deshidratación hipernatrémica

\begin{tabular}{|c|c|c|c|c|c|}
\hline Referencia & $\begin{array}{l}\text { Edad } \\
\text { en } \\
\text { días }\end{array}$ & $\begin{array}{l}\text { Nivel de } \\
\text { sodio sérico } \\
\text { (mmol/l) }\end{array}$ & Lugar de trombosis & Tratamiento & Estado final \\
\hline $\begin{array}{l}\text { Iglesias } \\
\text { Fernández, et al., } \\
2003^{3}\end{array}$ & 8 & 180 & $\begin{array}{l}\text { Seno venoso cerebral y } \\
\text { trombosis aórtica } \\
\text { abdominal }\end{array}$ & $\begin{array}{l}\text { Anticoagulación con } \\
\text { heparina y trombólisis } \\
\text { sistémica con alteplasa y } \\
\text { urocinasa }\end{array}$ & $\begin{array}{l}\text { Recanalización de la aorta } \\
\text { y el seno cerebral }\end{array}$ \\
\hline $\begin{array}{l}\text { Morales, et al., } \\
2008^{4}\end{array}$ & 9 & 171 & Aorta abdominal & $\begin{array}{l}\text { Trombólisis dirigida por } \\
\text { catéter, anticoagulación con } \\
\text { heparina y warfarina }\end{array}$ & $\begin{array}{l}\text { Recanalización aórtica y } \\
\text { amputación menor de } 3 \\
\text { dedos del pie }\end{array}$ \\
\hline $\begin{array}{l}\text { Hbibi, et al., } \\
2012^{5}\end{array}$ & 9 & 158 & $\begin{array}{l}\text { Seno venoso cerebral y } \\
\text { trombosis aórtica } \\
\text { abdominal }\end{array}$ & $\begin{array}{l}\text { Anticoagulación con } \\
\text { heparina y anticoagulantes } \\
\text { orales }\end{array}$ & $\begin{array}{l}\text { Recanalización aórtica y } \\
\text { venosa }\end{array}$ \\
\hline $\begin{array}{l}\text { Ayad, et al., } \\
2018^{6}\end{array}$ & 5 & 163 & $\begin{array}{l}\text { Aorta abdominal y renal } \\
\text { bilateral }\end{array}$ & Ninguno & Fallecido \\
\hline $\begin{array}{l}\text { Al Nuaimi, et al., } \\
2020^{2}\end{array}$ & 6 & 175 & $\begin{array}{l}\text { Trombosis aórtica } \\
\text { abdominal, inferior al } \\
\text { origen de la mesentérica } \\
\text { superior }\end{array}$ & $\begin{array}{l}\text { Trombólisis sistémica con } \\
\text { alteplasa y anticoagulación } \\
\text { sistémica con heparina }\end{array}$ & $\begin{array}{l}\text { Recanalización de aorta y } \\
\text { extremidades }\end{array}$ \\
\hline
\end{tabular}

Por parte de cardiología se realiza ecocardiograma identificando conducto arterioso permeable en vías de cierre, foramen oval permeable, función ventricular conservada, sin evidencia de vegetaciones 0 trombos.

Por parte de nuestro servicio se reportó que presenta perfusión tisular adecuada a nivel infragenicular, sin embargo, no presentó pulsos tibiales ni peroneo y solo flujos monofásicos al Doppler de onda continua.

Se realizó angiotomografía reportando oclusión aórtica parcial a nivel yuxtarrenal, con afectación de arteria renal izquierda, permaneciendo ocluida en su tercio proximal y recanalizando por colaterales, dos centímetros por debajo de arterias renales oclusión completa de aorta e ilíacas recanalizando hasta arterias femorales comunes, sin alteraciones distales (Fig. 5).

Se llevó a sala de operaciones, en conjunto con traumatología y ortopedia, posterior a cinco días de estancia en unidad de cuidados neonatales, con fines de laparotomía exploradora, trombectomía mecánica (Fig. 6); se abordó en línea media con una incisión supraumbilical e infraumbilical, haciendo disección con electrocauterio hasta localizar aorta y las dos arterias renales, por separado se refirieron con cintillas vasculares, se procede a realizar arteriotomía aórtica y posteriormente trombectomía mecánica, con catéter Fogarty 3 Fr, se encontraron coágulos de aspecto crónico, se obtuvo reflujo, se recuperaron pulsos femorales bilaterales, corroborados por visión directa y control con ultrasonido Doppler, asas intestinales de adecuada coloración, mesenterio sin alteraciones, por lo que se realizó arteriorrafia con prolene vascular 7-0, y se cerró por planos, finalizando acto quirúrgico.

Por parte de angiología, se encontró adecuada evolución, posteriormente a haber resuelto la deshidratación hipernatrémica y la oclusión arterial aguda, se anticoaguló con enoxaparina $1 \mathrm{mg}$ subcutánea cada 12 horas; sin embargo, fue egresado tras 10 días de estancia en la unidad de cuidados intensivos neonatales hasta la mejoría ventilatoria.

\section{Resultados}

Se realizó abordaje multidisciplinario descartando origen cardiaco o séptico. La evaluación del servicio de reumatología en búsqueda de patologías relacionadas con trombofilias está en evaluación en consultas subsecuentes, debido a la aparición de posibles falsos negativos relacionados con el proceso agudo intrahospitalario. En ambos casos se egresaron con antiagregación con ácido acetilsalicílico a dosis de $1 \mathrm{mg} / \mathrm{kg}$ cada 12 horas por 3 meses. 


\section{Discusión}

Los casos de trombosis aórtica espontánea son excepcionales, lo cual está relacionado con el sistema de hemostasia de los primeros días de vida. La trombosis aórtica es una complicación casi exclusiva de la cateterización de la arteria umbilical; ocurre con menor frecuencia que la trombosis venosa y cuando se presenta afecta más comúnmente arterias ilíacas, femorales y cerebrales. Existen en la literatura cinco casos reportados en la literatura de trombosis aórtica neonatal espontánea secundaria a deshidratación hipernatrémica de 2005 a 2020 (Tabla 2), sin embargo, existen más estudios de trombosis aórtica asociada a otras causas e incluso aunada a trombosis venosa. No se han reportado estadísticas en México con respecto a las trombosis aórticas neonatales, pero en un estudio de Dinamarca la tasa anual de trombosis arterial y venosa es $0.07 / 10,000$ niños, lo que representa $5.3 / 10,000$ niños hospitalizados y 24/10,000 recién nacidos ingresados en cuidados intensivos. Aproximadamente la mitad de las trombosis son arteriales, con mayor incidencia en el periodo neonatal $(2.3 / 10,000$ recién nacidos) ${ }^{7}$.

Para llevar a cabo el diagnóstico, cuando hay una trombosis instalada los signos y síntomas pueden ser tan obvios como: disminución de la temperatura, disminución de la perfusión de la piel y ausencia de pulsos en las extremidades; sin embargo, la presentación puede ser con hipertensión arterial, insuficiencia renal aguda o insuficiencia cardiaca congestiva, lo cual puede retrasar el diagnóstico ${ }^{6}$.

Para el diagnóstico de sospecha, además de la presentación de signos y síntomas, se usa ecografía Doppler, como método inicial, debido a que no es invasivo y es de bajo costo; para confirmarse se lleva a cabo la angiotomografía, que también es el mayor apoyo para planeación quirúrgica.

La terapia puede variar desde conservadora con anticoagulación, hasta trombólisis sistémica o trombectomía quirúrgica. El Colegio Americano de Médicos de Tórax recomienda el uso de primera línea de heparina no fraccionada o de bajo peso molecular, con una duración de cinco a siete días; la trombólisis se recomienda como segunda línea si falla la heparina o en trombosis arterial complicada; y en caso de que el pronóstico vital esté comprometido, trombectomía mecánica, aunque no ha establecido una pauta generalizada y el manejo varía dependiendo de cada centro médico ${ }^{8}$.
Por lo anteriormente descrito, se debe individualizar en cada paciente respecto a las comorbilidades, antecedentes clínicos y evolución perihospitalaria.

\section{Conclusión}

La trombosis aórtica en neonatos es un evento poco frecuente y con una importante morbimortalidad potencial. Para mejor pronóstico a corto plazo se requiere un alto índice de sospecha y pronta intervención por parte del cirujano vascular.

\section{Agradecimientos}

Los autores agradecen al personal que conforma el Servicio Quirúrgico del Instituto Mexicano del Seguro Social, en especial a la UMAE del Hospital de Pediatría de Guadalajara, Jalisco, por las facilidades brindadas para la realización de este trabajo.

\section{Financiamiento}

La presente investigación no ha recibido ninguna beca específica de agencias de los sectores públicos, comercial o sin ánimo de lucro

\section{Conflicto de intereses}

Los autores declaran no tener conflicto de intereses.

\section{Responsabilidades éticas}

Protección de personas y animales. Los autores declaran que para esta investigación no se han realizado experimentos en seres humanos ni en animales.

Confidencialidad de los datos. Los autores declaran que han seguido los protocolos de su centro de trabajo sobre la publicación de datos de pacientes.

Derecho a la privacidad y consentimiento informado. Los autores han obtenido el consentimiento informado de los pacientes y/o sujetos referidos en el artículo. Este documento obra en poder del autor de correspondencia.

\section{Bibliografía}

\footnotetext{
1. Nagel K, Tuckuviene R, Paes B, Chan AK. Neonatal aortic thrombosis: a comprehensive review. Klin Padiatr. 2010;222(3):134-9.

2. Al Nuaimi M, Williams S. Successful systemic thrombolysis in a neonatal occlusive abdominal aortic thrombus secondary to hypernatremic dehydration: A case report and literature review. J Pediatr Hematol Oncol. 2020;42(7):e589-e592.
} 
3. Iglesias Fernández C, Chimenti Camacho P, Vázquez López P, Guerrero Soler M, Blanco Bravo D. Trombosis aórtica y cerebral secundarias a deshidratación hipernatrémica en un recién nacido con lactancia materna exclusiva. An Pediatr (Barc.). 2003:65(4):381-3.

4. Morales JP, Sabharwal T, Tibby SM, Burnand KG. Successful thrombolysis of a symptomatic neonatal aortic thrombosis associated with hypernatraemic dehydration--case report and literature review. Int J Clin Pract. 2008:62(3):502-5.

5. Hbibi M, Abourazzak S, Babakhouya A, Boubou M, Atmani S, Tizniti S, et al. Severe hypernatremic dehydration associated with cerebral venous and aortic thrombosis in the neonatal period. BMJ Case Rep. 2012;2012:bcr0720114426.
6. Ayad A, Lemouakni S, Tami L, Oulja M, Knouni H, Barkat A. Thrombose aortique néonatale spontanée révélant un déficit en protéine $\mathrm{S}$. J Med Vasc. 2018;43(1):56-60.

7. Brice J, Venot $P$, Colinart-Thomas M, Morville P. Choc cardiogénique dû à une thrombose spontanée néonatale de l'aorte abdominale. Arch Pediatr. 2016;23(9):951-6.

8. Hirsh J, Guyatt G, Albers GW, Harrington R, Schünemann HJ. Antithrombotic and thrombolytic therapy: American College of Chest Physicians Evidence-Based Clinical Practice Guidelines (8 $8^{\text {th }}$ Edition). Chest. 2008;133(6 Suppl):110S-112S. 